\title{
Mapping perception of consumer antivirus software with multidimensional scaling method
}

\author{
Ai Nurhayati, Frencius \\ Sekolah Tinggi Teknologi Bandung \\ Soekarno-Hatta Street, (022) 5224000 \\ *Corresponding author, e-mail: ai.nurhayati@sttbandung.ac.id
}

\begin{abstract}
Antivirus software industry is growing rapidly in the world in 2018. The domestic antivirus software industry must be able to compete on a global scale. To face free trade, Indonesia's antivirus software industry must be able to know its position in the minds of consumers, especially domestic consumers. In this research, Smadav will represent the antivirus software industry from Indonesia. In this research want to know how the position of smadav compared with its current competitors, namely Avast, Avira, AVG, Kaspersky, McAfee and Norton. This research is only done to map antivirus software based on similarity according to respondent's perception. This research uses Multidimensional scaling (MDS) method through SPSS software program version 23. The results showed that there are three groups of different antivirus software based on similarity level according to the respondent's perception. On the two-dimensional and three-dimensional maps Norton antivirus software, Avast and Avira have similar resemblance according to the respondent's perception, because the location is closest and is in the same quadrant. Smadav differs according to perceptions of respondents. AVG, McAfee and Kaspersky have similarities according to respondents' perceptions.
\end{abstract}

Keywords: antivirus, mapping perception, multidimensional scaling(MDS), SPSS

Copyright $\odot 2019$ APTIKOM - All rights reserved.

\section{Introduction}

The rise of millions computer virus and malware has made programmer competing to create the best antivirus software, that can protect personal computer system and all of the files in it.

Antivirus software industry is growing rapidly in the world in 2018. The domestic antivirus software industry must be able to compete on a global scale. To face free trade, Indonesia's antivirus software industry must be able to know its position in the minds of consumers, especially domestic consumers.

From studies that have been available so far until 2018, it turns out that there is no research comparing Indonesian antivirus software products to foreign products. So the research here is directed to examine the similarity of Indonesian antivirus software products to foreign products based on consumer perceptions. So from this research it is expected that Indonesian antivirus software products can meet the criteria of products desired by consumers.

The problem that will be examined from this research is the position of domestic antivirus software compared to antivirus software from abroad according to consumer perceptions. In this research, Smadav will represent the antivirus software industry from Indonesia. Smadav will be examined its position in the minds of consumers with its competitors, namely Avast, Avira, AVG, Kaspersky, McAfee and Norton.

The purpose of this research is to find out the position of domestic antivirus software compared to antivirus software from abroad according to consumer perceptions. The purpose of this study is to determine the position of Smadav in the minds of consumers when compared with competitors according to the perceptions of the respondents. Benchmarking will be done by perceptual map. This study is only done to map antivirus software based on similarity according to respondent's perception.

This research uses multidimensional scaling (MDS) method because MDS can describe the position of an object with another object based on the similarity of the objects. This research was conducted with SPSS version 23 software program because SPSS was able to model using ALSCAL and INDSCAL methods. 


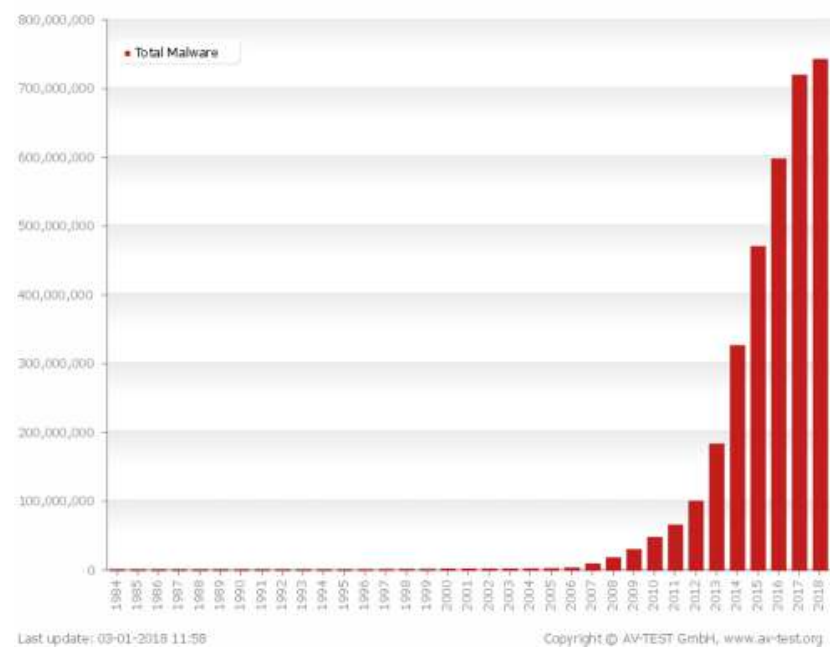

Figure 1. Total malware [1]

\section{Research Method}

This research uses Multidimensional scaling method through SPSS version 23 software program. Multidimensional Scaling is techniques for constructing a map of generally high-dimensional data into a target dimension with respect to the given pairwise proximity information [2]. Multidimensional Scaling is related to the creation of a map to describe the position of an object with another object based on similarity of those objects. This research is done with SPSS version 23 software program because SPSS able to make model using ALSCAL and INDSCAL method.

Multidimensional Scaling describes analysis of proximity data on a set of stimuli to reveal the hidden structure underlying the data [3]. The proximity data come from measure of pairwise similarity [4]. Based on a proximity matrix measured on objects as input entity, these distances are mapped on a lower dimensional spatial [5]. The goal of Multidimensional Scaling is to find low-dimensional reconstruction vectors $y_{i}$ that minimize the following function [6]:

$$
\varphi(y)=\sum_{(i, j)}\left(d_{i j}-\left\|y_{i}-y_{j}\right\|\right)^{2}
$$

where $d_{i j}=d_{j i}$ is the Euclidian distance between the original data points and $\left\|y_{i}-y_{j}\right\|$ denotes the Euclidian distance in the reconstruction space. The research steps in the form of Multidimensional Scaling decision diagram are presented in Figure 2 [7]. 

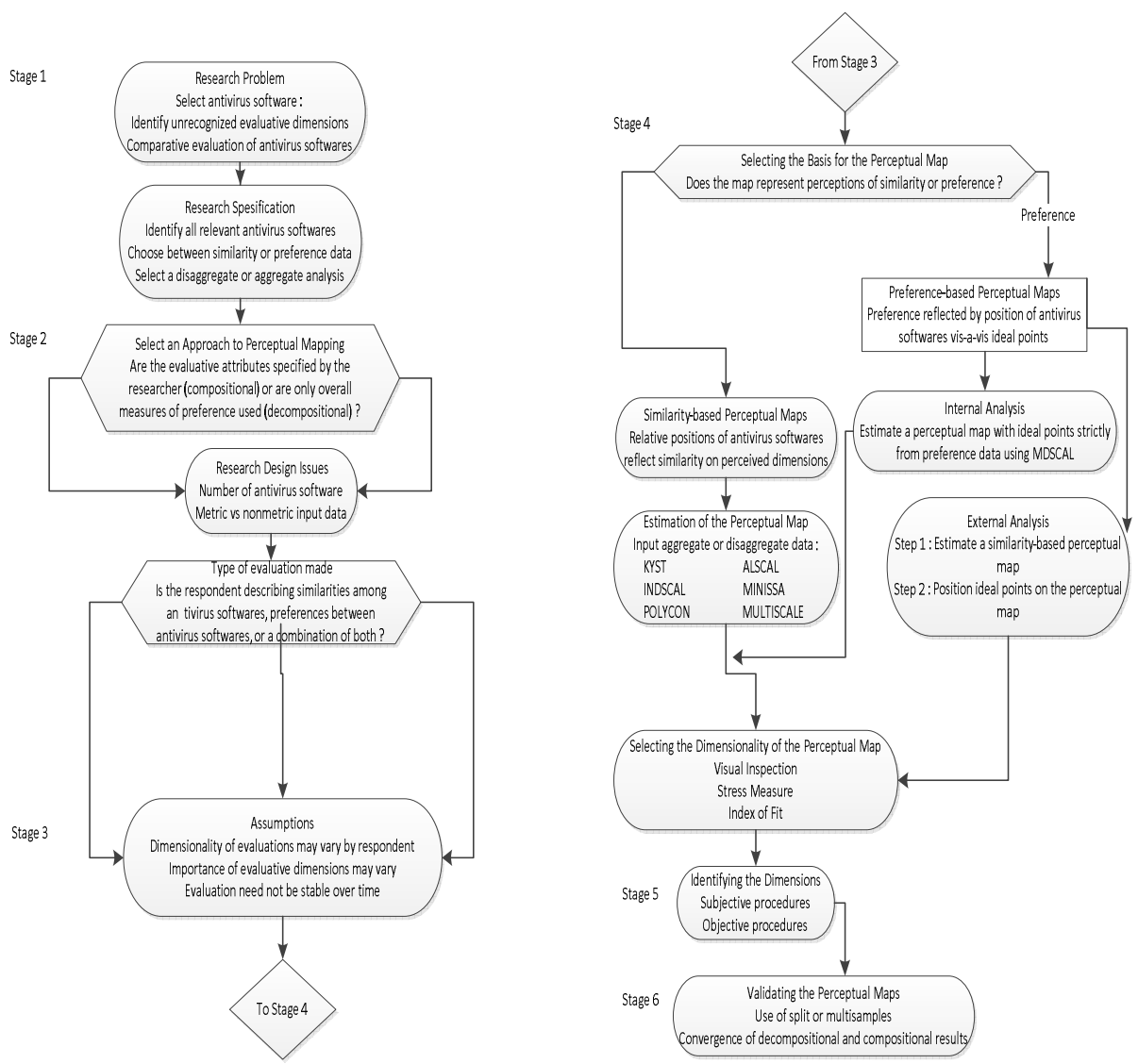

Figure 2. Multi dimensional scaling (MDS) decision diagram

Data obtained from the distribution of questionnaires to many respondents who have been frequently using antivirus software and is considered to reflect consumer attitudes so far.

The respondents were asked to provide an assessment of the similarity between smadav and other brands, on a scale of 1 to 5 , in which:

a. Scale 1 means two distinct brands that compare very closely

b. Scale 5 means two particular brands that are compared very unlike (different)

Respondents can assign values between scales of 1 to 5 .

Test the alignment of respondents in the assessment is available in software program SPSS version 23.

\section{Results and Analysis}

This research is different from other studies because most of the existing research only lists antivirus software products originating from abroad, there is no research that includes Indonesian products. For example, a paper written by Vaclav Bezdec entitled "Comparison of Anti-Virus Programs using Fuzzy Logic" [8], the aim is only to choose the best anti-virus software based on testing made by AVComparatives, so it is not based on consumer perceptions. Consumer perception is very important because the number of products sold is caused by consumer perceptions. In this study included Indonesian antivirus products compared to foreign products based on consumer perceptions. Consumer perception mapping on antivirus software using multidimensional scaling methods with SPSS. Below is the output of SPSS with a multidimensional scaling method consisting of two-dimensional and three-dimensional images.

\subsection{Two Dimension}

The Figure 3 is the mapping from INDSCAL process to display the Multidimensional Scaling from antivirus software case. It's seen that Norton antivirus software has some similarities with Avira and Avast from their position in the map. The other group is AVG, McAfee and Kaspersky that are located near 
to each other. Thus AVG, McAfee and Kaspersky have some similarities. Smadav is located far away from the two groups. This antivirus is the most differentiated from the others. Respondent similarity test in assessment is presented in the Figure 4. On the graph in Figure 4, it is seen that the respondent dot position can be made into a line that pointing to the right. This prove that there is a consistency on the respondent in assessing the similarities from 7 antivirus software (Smadav, Avast, Avira, AVG, Kaspersky, McAfee and Norton). In this graph (Figure 5) that consist of dots coordinate from the 26x7 questionnaire, it's seen that the dots are located relative in the centre of the graph. This prove the similarities of attitude from the respondent.

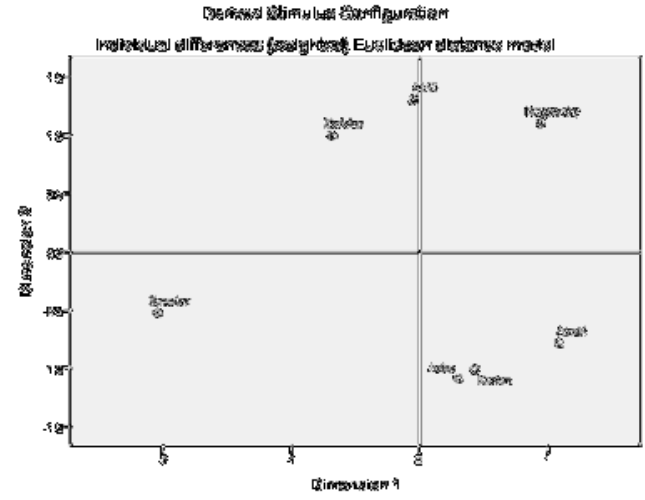

Figure 2. Derived Stimulus Configuration Individual differences (weighted) Euclidean distance model

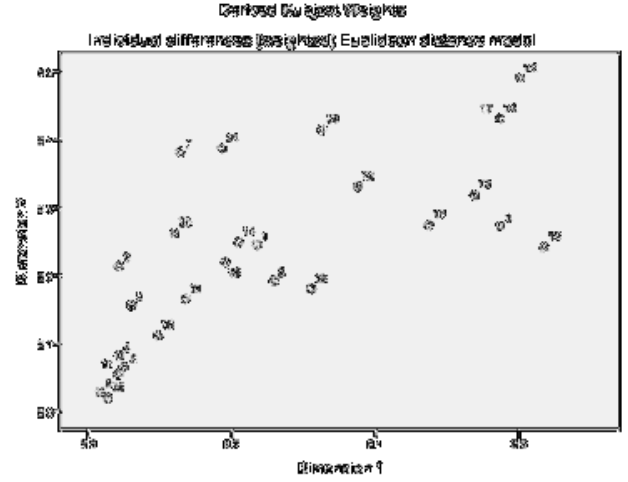

Figure 3. Derived subject weights individual differences (weighted) Euclidean distance model

\subsection{Three dimension}

Figure 6 is the map from INDSCAL process to display Multi Dimensional Scaling map in three dimension. It is seen that antivirus software Avast is similar to Avira and Norton. AVG antivirus similar to McAfee and Kaspersky. While smadav is different from all of the other antivirus.

From 7 antivirus software, it turns out that it can be divided into 3 groups based on similarities according to consumer perceptions. Group 1 consists of: Avast, Avira and Norton. Group 2 consists of: AVG, McAfee and Kaspersky. Group 3 consists of: Smadav. The division of this group is not based on the best antivirus performance, but the division of this group is only based on product similarity in mind from consumers in Indonesia who have been statistically tested with multidimensional scaling methods.

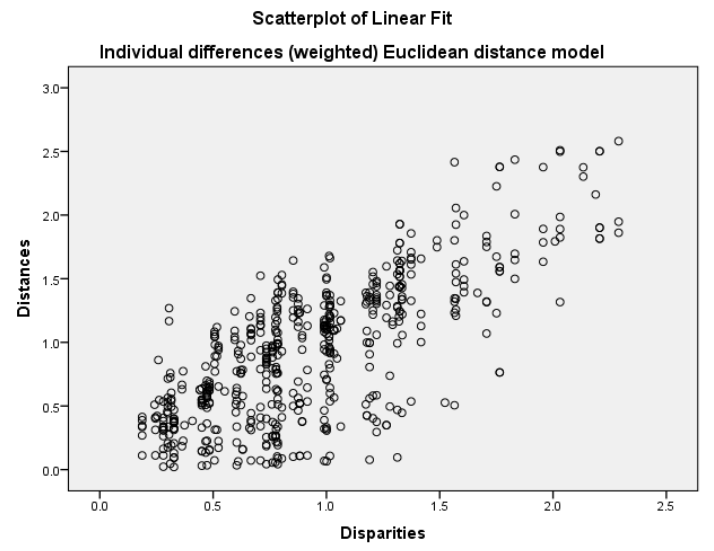

Figure 4. Scatterplot of linear fit individual differences (weighted) Euclidean distance model

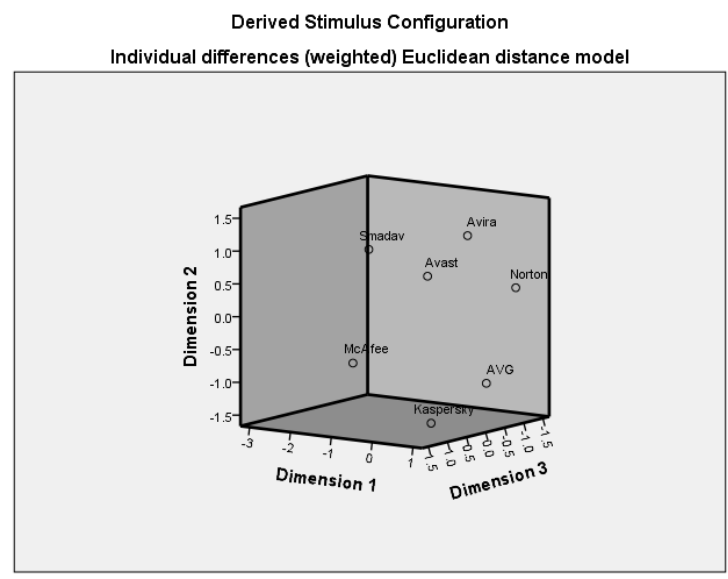

Figure 5. Derived stimulus configuration individual differences (weighted) Euclidean distance model

APTIKOM J. CSIT Vol. 4, No. 3, 2019: $91-95$ 


\section{Conclusion}

On the two-dimensional and three-dimensional maps Norton antivirus software, Avast and Avira have similar resemblance according to the respondent's perception, because the location is closest and is in the same quadrant. Smadav differs according to perceptions of respondents. AVG, McAfee and Kaspersky have similarities according to respondents' perceptions. In the next research can be developed various marketing strategies based on the results perceptual map.

\section{References}

[1] www.av-test.org

[2] Bae S H. Scalable High Performance Multidimensional Scaling. Disertation. Indiana University; 2012.

[3] Steyvers M. Multidimensional Scaling. Encyclopedia of Cognitive Science. 2002; 1.

[4] Izenman A. Modern Multivariate Statistical Techniques Regression, Classification, and Manifold Learning. Philadelphia: Springer. 2013: 464.

[5] Leeuw J, Mair P. Multidimensional Scaling Using Majorization: SMACOF in R. Journal of Statistical Software. year; Vol. 31(3): 1.

[6] Otty T, Sch"uley M, Heldz J, Albertz C and Stoop R. Clustered Multidimensional Scaling with Rulkov Neurons. International Symposium on Nonlinear Theory and Its Applications, NOLTA. Japan. 2016: 389-390.

[7] Hair J F, Black W C, Babin B J, Anderson R E. Multivariate Data Analysis: Pearson. 2010: 573-595.

[8] Bezdec V. Comparison of Anti-Virus Programs using Fuzzy Logic. Journal of Systems Integration; 2013. 